\title{
Specific Dysphoric Symptoms Are Predicted by Early Maladaptive Schemas
}

\author{
Roberta Trincas, ${ }^{1}$ Cristina Ottaviani, ${ }^{2,3}$ Alessandro Couyoumdjian, ${ }^{3}$ Katia Tenore, \\ Grazia Spitoni, ${ }^{2,3}$ and Francesco Mancini ${ }^{1}$ \\ ${ }^{1}$ Scuola di Psicoterapia Cognitiva S.r.l., Viale Castro Pretorio 116, 00185 Roma, Italy \\ ${ }^{2}$ IRCCS Santa Lucia Foundation, Via Ardeatina 306, 00142 Rome, Italy \\ ${ }^{3}$ Department of Psychology, Sapienza University of Rome, Via dei Marsi 78, 00185 Rome, Italy
}

Correspondence should be addressed to Roberta Trincas; trincas.roberta@gmail.com

Received 18 October 2013; Accepted 10 December 2013; Published 8 January 2014

Academic Editors: C. M. Beasley, C. Evren, R. R. Tampi, and A. J. Torres

Copyright (C) 2014 Roberta Trincas et al. This is an open access article distributed under the Creative Commons Attribution License, which permits unrestricted use, distribution, and reproduction in any medium, provided the original work is properly cited.

\begin{abstract}
Early maladaptive schemas (EMSs) are cognitive patterns resulting from unmet core emotional needs in childhood that have been linked to the development of psychopathology. As depression is a multifaceted phenomenon, we hypothesized that specific dysphoric symptoms would be predicted by different EMSs. Four hundred and fifty-six participants completed a measure of EMSs (Young Schema Questionnaire) and reported on the severity of the symptoms of criterion A for major depression in DSM-IV during the occurrence of a dysphoric episode in the previous 12 months. A series of stepwise multiple regression analyses were performed to investigate the predictive power of the EMSs for the severity of each specific depressive symptom. When controlling for gender and current levels of depression, specific symptoms were predicted by different EMSs: sadness by Negativity/Pessimism; anhedonia by Failure; self-harm by Emotional Deprivation and Vulnerability to Harm or Illness; worthlessness by Failure and Negativity/Pessimism; psychomotor retardation/restlessness by Vulnerability to Harm or Illness and Entitlement/Grandiosity; and poor concentration by Insufficient Self-Control/Self-Discipline. The more physical symptoms of fatigue, insomnia/hypersomnia, and appetite loss/appetite gain were not predicted by any of the EMSs. Although the cross-sectional design of the study does not allow for conclusions about the direction of effects, results suggest that depression is not a unitary phenomenon and provide a possible explanation for previous inconsistent findings.
\end{abstract}

\section{Introduction}

In recent years, the view that depression is not a unitary phenomenon has received much support and several studies demonstrated the possibility to identify different kinds of depressive reactions associated with specific types of adverse life events $[1,2]$. According to cognitive theories of depression [3], it is not the negative event itself but the way the individual interprets the event that leads (or not) to depressive symptoms [4]. As a consequence, when a stressful life event occurs, individuals who have negative cognitive schemas or core beliefs are at an increased risk for depression.

The Young Schema Theory [5] expands on Beck's theory, offering an explanation of the developmental origins of these cognitive factors. According to the theory, individuals who experience toxic events during childhood are prone to develop dysfunctional character traits called "early maladaptive schemas" (EMSs). Young et al. [5] identified 18 EMSs and developed an ad hoc instrument for their assessment: the Young Schema Questionnaire [6]. To date, this self-report measure has been mainly used to study the relationship between EMSs and personality disorders. In the past 15 years, however, the schema model has been also shown to be relevant to mood disorders [7]. Studies have been conducted on both clinical and nonclinical samples, showing that people with mood disorders tend to have higher scores on most or all EMSs compared to healthy controls [8]. In general, findings suggest that EMSs are underlying character traits that remain stable over time rather than a simple reflection of symptoms $[9,10]$. For example, history of depression is associated with higher EMSs even in the absence of current depressive symptoms [11], and depressed mood induction 
in a nonclinical sample had no significant effect on EMSs [12].

The few studies relating specific schema domains and depression, however, found a wide and inconsistent range of EMSs [7]. For example, Calvete and colleagues [13] found that the EMSs failure, defectiveness/shame, and self-sacrifice were associated with depressive symptom severity in non-clinically depressed samples. Another study showed that the EMSs Defectiveness/Shame, Insufficient Self-Control, Vulnerability, and Incompetence/Inferiority were cross-sectionally related to depressive symptom severity in undergraduate students [14]. Schmidt and colleagues [15] found that the original Dependency and Defectiveness/Shame EMSs accounted for $33 \%$ of the variance of depression in a nonclinical sample. Calvete et al. [13] reported that the Defectiveness/Shame, Insufficient Self-Control, Incompetence, and Vulnerability to Harm or Illness EMSs explained 63\% of the variance of selfreported depressive symptoms while in Stopa et al. [16] the EMSs Abandonment, Defectiveness/Shame, Subjugation, and Self-Sacrifice explained $43 \%$ of the variance of depression. In Welburn and colleagues [17], 12 of 15 assessed EMSs were significantly correlated with the symptoms of depression, but only the Abandonment and Insufficient Self-Control EMSs made a unique contribution to the prediction of depression.

A possible explanation for such inconsistencies comes from the use of different samples, such as groups with a wide range of comorbidities, mixed clinical samples (mood and anxiety disorders), and nonclinical groups [9, 13-17]. In fact, when samples with different psychopathological conditions (e.g., chronic and nonchronic depression and bulimia nervosa) were compared, results showed differences in their EMSs $[18,19]$.

Here, we propose that another possible explanation for such contradictory findings may derive from the simplistic view of depression as a unitary phenomenon. In line with a dimensional perspective of psychopathology that postulates the existence of a continuum from low mood to clinical depression, we investigated dysphoric reactions after a stressful episode in healthy individuals [20]. We specifically hypothesized that certain EMSs would be associated with different symptoms of depression. According to cognitive theory [21], we assumed that when a stressful life event occurs, specific schemas are activated that affect the way the individual interprets the event, leading to specific depressive symptoms.

\section{Methods}

2.1. Participants. The data are part of a larger study investigating cognitive factors in dysphoric episodes [4]. The study was conducted through an online survey, and participants were recruited via advertisements on the internet. Seven hundred and eighty-nine participants agreed to participate and 511 completed the study (64.8\%). In all, 456 (89.2\%) reported the occurrence of a dysphoric episode (i.e., a 5-day period of low mood) during the previous 12 months. The sample consisted of 328 women (mean age $=38.8 .1(1.5)$ years) and 128 men (mean age $=42.7$ (9.7) years). All subjects were Caucasians. Fifty-six subjects $(12.3 \%)$ had a past diagnosis of depression,
$80(17.5 \%)$ took antidepressants in the past, and 36 (7.9\%) were taking psychiatric medications other than antidepressants at the time of the study. The majority of the participants were in paid employment $(n=397)$ and had completed a higher vocational or university education $(n=364)$.

2.2. Procedure. The survey was administered in a single session by Questionpro.com, which guarantees the privacy and confidentiality of the respondents, and it took about $45 \mathrm{~min}$ to complete. As the online administration provided us with a measure of the time taken to fill out the questionnaire, we were able to exclude outliers (less than $2 \%$ of the respondents). After providing instructions and informed consent, all respondents completed a series of forms in the same order as presented below. After survey completion, participants were debriefed and thanked for their time. Debriefing consisted of a brief explanation of the background and aim of the study and feedback of subjects' personal scores at standardized questionnaires. The study was conducted according to the Declaration of Helsinki guidelines.

2.3. Sociodemographic and Personal Information. After providing instructions and informed consent, participants were asked to complete a sociodemographic form that included items regarding age, gender, education, and employment.

2.4. Questionnaires. Following the methodology described in Keller et al. [1], we assessed the occurrence of the worst dysphoric episode over the previous year, asking subjects to indicate one or more symptoms experienced in the past year. To do so, we presented a list of twelve symptoms extracted from criterion A for major depression in DSM-IV (sadness, anhedonia, fatigue, psychomotor retardation/restlessness, insomnia/hypersomnia, appetite loss/appetite gain, self-harm, poor concentration, and worthlessness) for at least 5 days. Participants were asked to signal how much each symptom interfered with their daily life (from 0 , absence of the symptom, to 4 , symptom interfering completely with daily life). If they did not experience a dysphoric episode in the previous year (i.e., they scored 0 to all symptoms), they automatically exited the survey. Then, we asked if something happened that might have contributed to make them feel that way, and possible answers were (a) no, I cannot identify any particular event and (b) yes, the symptoms are the consequences of particular events. When the answer was negative, participants were associated with the "no specific cause" category; when it was affirmative, they were asked to further identify the plausible reason for this period of low mood (ALE), first describing it in a free-format paragraph and then selecting the single most likely cause from Keller et al. [1] categories (failure, health problems, interpersonal conflict, death of a loved one, romantic breakup, stress, scare, and others).

2.5. Young Schema Questionnaire-Short Form 3. The YSQ-S3 [6] is a 90-item questionnaire that assesses 18 EMSs. Each scale consists of five items, and participants are asked to rate the items using a 6-point Likert scale $(1=$ completely untrue of me, 2 = mostly untrue of me, 3 = slightly more untrue than 
true, $4=$ moderately true of me, $5=$ mostly true of me, and 6 = describes me perfectly). The 18 EMSs are (1) Abandonment, (2) Mistrust/Abuse, (3) Emotional Deprivation, (4) Defectiveness/Shame, (5) Social Isolation/Alienation, (6) Dependence/Incompetence, (7) Vulnerability to Harm or Illness, (8) Enmeshment/Undeveloped Self, (9) Failure, (10) Entitlement/Grandiosity, (11) Insufficient Self-Control/Self-Discipline, (12) Subjugation, (13) Self-Sacrifice, (14) Approval-Seeking/Recognition-Seeking, (15) Negativity/Pessimism, (16) Emotional Inhibition, (17) Unrelenting Standards/Hypercriticalness, and (18) Punitiveness. In this study, the average of the item scores was computed for each schema (range $=1$ to 6 ), as this method has been reported and indicated as the most reliable in discriminating between clinical and nonclinical groups $[19,22]$.

2.6. Center for Epidemiologic Studies Depression Scale. To assess current depressive state, we administered the Italian version of the Center for Epidemiologic Studies Depression Scale (CES-D) [23] which has shown good reliability and convergent validity with related self-report measures. The CES$\mathrm{D}$ is a 20-item self-report scale that assesses the frequency of occurrence of symptoms of depression during the past week. Total score ranges from 0 to 60 . Standard cut-offs are $>16$ for mild depression and $>23$ for clinical depression.

2.7. Statistical Analysis. All data are expressed as means (SD). Data analyses were performed with Statistica 8 (StatSoft. Inc., USA).

First, the intercorrelations between the different EMSs were computed. To investigate the predictive power of the YSQ dimensions for the occurrence of specific depressive symptoms during a past dysphoric episode, nine forward stepwise multiple regression analyses were performed, with each symptom (sadness, anhedonia, fatigue, psychomotor retardation/restlessness, insomnia/hypersomnia, appetite loss/appetite gain, self-harm, poor concentration, and worthlessness) as the dependent variable. Due to the number of EMSs and hence comparisons, the $P$-to-enter was Bonferroni adjusted ( $P=0.0025-0.05 / 20$ comparisons). To control for gender and current level of depression (CES-D score), these variables were always entered as predictors in the models.

\section{Results}

Table 1 shows means and standard deviations for the variables of interest. The average time from the event was 7.3 (4.5) months. Table 2 shows means and standard deviations for each EMS in men and women. Men had higher levels of Social Isolation/Alienation $(P=0.01)$ and Emotional Inhibition $(P=0.002)$ and women had higher levels of Enmeshment/Undeveloped Self $(P=0.02)$ and Self-Sacrifice $(P=$ 0.004).

Scores of the different EMSs were intercorrelated (see Table 3), except for the absence of correlation between (1) Self-Sacrifice and the EMSs Abandonment, Social Isolation, Defectiveness/Shame, Failure, Dependence/Incompetence, Vulnerability to Harm or Illness, Emotional Inhibition,
TABLE 1: Means and standard deviations for the current level of depression (range 0-57) and the symptoms (range 0-4) at the time of the dysphoric episode.

\begin{tabular}{lc}
\hline & $M(\mathrm{SD})$ \\
\hline CES-D & $16.1(11.2)$ \\
Sadness & $3.1(1.3)$ \\
Anhedonia & $2.4(1.4)$ \\
Fatigue & $2.7(1.2)$ \\
Psychomotor retardation/Restlessness & $1.7(0.9)$ \\
Insomnia/Hypersomnia & $2.1(0.9)$ \\
Appetite loss/Appetite gain & $1.9(0.8)$ \\
Self-harm & $1.9(1.2)$ \\
Poor concentration & $2.5(1.3)$ \\
Worthlessness & $2.3(1.3)$ \\
\hline
\end{tabular}

TABLE 2: Mean EMS scores.

\begin{tabular}{lccc}
\hline & Men & Women & $t$ \\
\hline Abandonment & $2.1(1.1)$ & $2.2(1.1)$ & 1.0 \\
Mistrust/Abuse & $2.2(0.9)$ & $2.2(1.0)$ & -0.3 \\
Emotional Deprivation & $1.8(0.9)$ & $1.9(1.1)$ & 0.9 \\
Defectiveness/Shame & $1.6(0.9)$ & $1.5(1.0)$ & -0.6 \\
Social Isolation/Alienation & $2.4(1.2)$ & $2.1(1.1)$ & $-2.6^{*}$ \\
Dependence/Incompetence & $1.6(0.7)$ & $1.5(0.7)$ & -1.7 \\
Vulnerability to Harm or Illness & $1.6(0.7)$ & $1.8(0.9)$ & 1.7 \\
Enmeshment/Undeveloped Self & $1.6(0.7)$ & $1.8(0.9)$ & $2.3^{*}$ \\
Failure & $1.7(0.9)$ & $1.7(1.0)$ & 0.2 \\
Entitlement/Grandiosity & $2.7(1.0)$ & $2.6(0.9)$ & -0.7 \\
Insufficient Self-Control/Self-Discipline & $2.4(1.1)$ & $2.2(0.9)$ & -1.9 \\
Subjugation & $1.9(0.9)$ & $1.9(0.9)$ & -0.2 \\
Self-Sacrifice & $2.8(0.9)$ & $3.1(1.1)$ & $2.9^{*}$ \\
Approval-Seeking/Recognition-Seeking & $2.8(1.0)$ & $2.6(1.0)$ & -1.9 \\
Negativity/Pessimism & $2.1(1.0)$ & $2.1(1.0)$ & 0.1 \\
Emotional Inhibition & $2.7(1.2)$ & $2.4(1.1)$ & $-3.1^{*}$ \\
Unrelenting Standards/ & $3.3(0.9)$ & $3.3(0.9)$ & 0.1 \\
Hypercriticalness & $2.3(1.0)$ & $2.2(1.0)$ & -0.6 \\
Punitiveness & & &
\end{tabular}

Note. ${ }^{*} P<0.05$.

Insufficient Self-Control/Self-Discipline, Approval-Seeking/ Recognition-Seeking, Negativity/Pessimism, and (2) Entitlement/Grandiosity and Failure. In regard to multicollinearity, the present data showed no minimal tolerance problem for the predictors.

Results from the stepwise multiple regression analyses (see Table 4) showed that the current level of depression was significantly associated with all the symptoms experienced at the time of the dysphoric episode. In line with our hypothesis, different EMSs were related to specific symptoms. The symptom of sadness was significantly predicted by Negativity/Pessimism $\left(R^{2}=0.38 ; P<0.0001\right)$; anhedonia was predicted by Failure $\left(R^{2}=0.42 ; P<0.0001\right)$; self-harm was predicted by Emotional Deprivation and Vulnerability to 
TABLE 3: Intercorrelations between EMSs.

\begin{tabular}{|c|c|c|c|c|c|c|c|c|c|c|c|c|c|c|c|c|c|c|}
\hline & 1 & 2 & 3 & 4 & 5 & 6 & 7 & 8 & 9 & 10 & 11 & 12 & 13 & 14 & 15 & 16 & 17 & 18 \\
\hline (1) Abandonment & * & & & & & & & & & & & & & & & & & \\
\hline (2) Mistrust/Abuse & 0.55 & * & & & & & & & & & & & & & & & & \\
\hline (3) Emotional Deprivation & 0.43 & 0.56 & * & & & & & & & & & & & & & & & \\
\hline (4) Defectiveness/Shame & 0.55 & 0.53 & 0.56 & $*$ & & & & & & & & & & & & & & \\
\hline (5) Social Isolation & 0.48 & 0.54 & 0.57 & 0.67 & * & & & & & & & & & & & & & \\
\hline (6) Dependence & 0.51 & 0.30 & 0.26 & 0.56 & 0.47 & $*$ & & & & & & & & & & & & \\
\hline (7) Vulnerability to Harm & 0.44 & 0.42 & 0.27 & 0.46 & 0.40 & 0.45 & $*$ & & & & & & & & & & & \\
\hline (8) Undeveloped Self & 0.38 & 0.33 & 0.25 & 0.27 & 0.32 & 0.45 & 0.36 & * & & & & & & & & & & \\
\hline (9) Failure & 0.43 & 0.29 & 0.26 & 0.56 & 0.45 & 0.70 & 0.44 & 0.38 & * & & & & & & & & & \\
\hline (10) Entitlement/Grandiosity & 0.36 & 0.44 & 0.34 & 0.25 & 0.43 & 0.24 & 0.26 & 0.25 & 0.12 & * & & & & & & & & \\
\hline (11) Insufficient Self-Control & 0.44 & 0.32 & 0.28 & 0.41 & 0.41 & 0.55 & 0.37 & 0.35 & 0.52 & 0.47 & * & & & & & & & \\
\hline (12) Subjugation & 0.58 & 0.49 & 0.50 & 0.58 & 0.58 & 0.57 & 0.48 & 0.50 & 0.54 & 0.36 & 0.55 & $*$ & & & & & & \\
\hline (13) Self-Sacrifice & 0.15 & 0.27 & 0.20 & 0.03 & 0.07 & 0.04 & 0.12 & 0.29 & 0.06 & 0.22 & 0.09 & 0.22 & $*$ & & & & & \\
\hline (14) Approval-Seeking & 0.46 & 0.35 & 0.21 & 0.35 & 0.30 & 0.41 & 0.32 & 0.30 & 0.35 & 0.41 & 0.48 & 0.45 & 0.08 & $*$ & & & & \\
\hline (15) Negativity/Pessimism & 0.58 & 0.55 & 0.35 & 0.53 & 0.47 & 0.50 & 0.76 & 0.39 & 0.50 & 0.32 & 0.44 & 0.56 & 0.13 & 0.44 & $*$ & & & \\
\hline (16) Emotional Inhibition & 0.36 & 0.38 & 0.35 & 0.43 & 0.50 & 0.29 & 0.29 & 0.26 & 0.30 & 0.28 & 0.24 & 0.45 & 0.09 & 0.24 & 0.32 & $*$ & & \\
\hline (17) Unrelenting Standards & 0.23 & 0.33 & 0.23 & 0.28 & 0.40 & 0.24 & 0.34 & 0.24 & 0.22 & 0.41 & 0.18 & 0.34 & 0.32 & 0.31 & 0.39 & 0.35 & $*$ & \\
\hline (18) Punitiveness & 0.35 & 0.41 & 0.28 & 0.44 & 0.42 & 0.36 & 0.46 & 0.23 & 0.41 & 0.26 & 0.30 & 0.41 & 0.19 & 0.31 & 0.56 & 0.29 & 0.53 & * \\
\hline
\end{tabular}

TABle 4: Final steps $(P$-to-enter $=0.0025)$ of the stepwise regression models of EMSs predicting symptoms scores $(n=456)$.

\begin{tabular}{lcccc}
\hline Dep var & Predictors & $B$ & SE & \\
Sadness & Gender & 0.18 & 0.05 & 0.12 \\
& CES-D & 0.06 & 0.01 & 0.06 \\
Anhedonia & Negativity/Pessimism & 0.19 & 0.01 & 0.15 \\
& CES-D & 0.07 & 0.06 & 0.01 \\
Self-Harm & Failure & 0.23 & 0.16 \\
& CES-D & 0.03 & 0.05 & 0.31 \\
& Emotional Deprivation & 0.16 & 0.06 \\
Worthlessness & Vulnerability to Harm & 0.45 & 0.01 \\
& CES-D & 0.04 & 0.06 \\
Psychom/Restlessness & Failure & 0.36 & 0.06 \\
& Negativity/Pessimism & 0.25 & 0.31 \\
Poor concentration & CES-D & 0.03 & 0.01 \\
& Vulnerability to Harm & 0.19 & 0.05 \\
\end{tabular}

Note. $B$ : unstandardized regression coefficient; SE: standard error; $\beta$ : standardized regression coefficient.

Harm or Illness $\left(R^{2}=0.37 ; P<0.0001\right)$; worthlessness was predicted by Failure and Negativity/Pessimism $\left(R^{2}=0.45 ; P<\right.$ $0.0001)$; Psychomotor retardation/Restlessness was predicted by Vulnerability to Harm or Illness and Entitlement/ Grandiosity $\left(R^{2}=0.25 ; P<0.0001\right)$; poor concentration was predicted by Insufficient Self-Control/Self-Discipline $\left(R^{2}=\right.$ $0.40 ; P<0.0001)$. Occurrence of the more physical symptoms of fatigue, insomnia/hypersomnia, and appetite loss/ appetite gain was only predicted by the CES-D scores $\left(R^{2}=\right.$ $0.14 ; P<0.0001$ for appetite; $R^{2}=0.24 ; P<0.0001$ for fatigue; $R^{2}=0.24 ; P<0.0001$ for sleep) but not by the EMSs.

\section{Discussion}

In the Diagnostic and Statistical Manual of Mental Disorders, 4th Edition, Text Revision (DSM-IV-TR; 2000), distinct subtypes based on symptomatology and its determinants are taken into account for anxiety disorders, while depression is only classified according to temporal criteria. The present study was theoretically driven by recent findings on the existence of different depressive subtypes in response to certain life events $[1,4,24,25]$. Specifically, we aimed at exploring if symptoms of depression that emerged during a dysphoric episode after a stressful event could be predicted 
by stable personality characteristics such as the EMSs. Results supported the hypothesis and provided a possible explanation for previous contradictory findings. Our study differs from previous research for two main aspects: first, we considered the specific symptoms of the depressive symptomatology instead of the categorical diagnosis or the severity of depression and second, we considered dysphoric episodes that occurred after a stressful event. The latter aspect supports the assumptions of the Schema Therapy model [5], implying that EMS may remain relatively latent until activated by stressful life events.

First, results showed that the occurrence of sadness after a stressful event was predicted by the EMS Negativity/Pessimism, that is, the pervasive focus on the negative aspects of life. Interestingly, despite the reasonableness of such association, this EMS has never been linked to depression. In our opinion, this is the consequence of a reductive and oversimplified view of depression that merges symptoms that are totally different and not necessarily present together.

Second, the occurrence of anhedonia was predicted by the EMS Failure, that is, the belief that one is inadequate compared with others. Accordingly, the diathesis-stress model of depression [26] hypothesizes the existence of sociotropic and autonomic individuals characterized by different depressive reactions: the first more likely to show depressive symptoms following interpersonal loss experiences, the second more vulnerable to events of failure. Our results make a step further suggesting the association between the EMS Failure and a specific depressive symptom such as anhedonia. This finding is also in agreement with Calvete et al. [13], who found that this EMS predicted depressive symptoms among 407 undergraduate students.

Self-harm was predicted by Emotional Deprivation, (i.e., the feeling that adequate emotional support is not available) and Vulnerability to Harm or Illness (i.e., the belief that catastrophe is impending). Emotional Deprivation has been previously associated with depressive symptom severity [27]. Moreover, Vulnerability to Harm or Illness, together with Defectiveness/Shame and Insufficient Self-Control, has been shown to explain $63 \%$ of the variance of self-reported depressive symptoms [14]. These results are also consistent with a study in which the EMSs Emotional Deprivation, Dependence/Incompetence, and Vulnerability to Harm were able to differentiate between suicidal depressed, non-suicidaldepressed, and nonclinical samples [28]. It has to be noted that the EMS Vulnerability to Harm or Illness, together with Entitlement/Grandiosity, predicted the occurrence of psychomotor retardation/restlessness. It is intriguing that, EMS Entitlement/Grandiosity has been previously considered a signature of bipolar disorder [29].

The symptom of worthlessness was predicted by Failure and Negativity/Pessimism (i.e., the pervasive focus on negative aspects of life). Again, only Failure has been previously associated with depression [13].

Finally, poor concentration was predicted by Insufficient Self-Control/Self-Discipline, that is the belief that one cannot restrain emotions or impulses. This association is not surprising if we consider that items are, for example: "When tasks become difficult, I usually cannot persevere and complete them." Moreover, Insufficient Self-Control appeared to be a key EMS specific to the symptoms of depression and to MDD in several studies [11, 13-17, 30, 31].

With regard to gender, the only studies that specifically examined differences between men and women in EMSs have been conducted on clinical populations. In a group of opioid users, Shorey et al. [32] showed that women scored significantly higher than men on three schema domains, including disconnection and rejection, impaired autonomy, and other directedness. In line with this result, we found higher levels of Enmeshment/Undeveloped Self and Self-Sacrifice in women. Studies on gender differences in mood disorders showed that women tend to be less assertive, more prone to helplessness, and more likely to overvalue relationships as sources of selfworth, which are all risk factors contributing to depression [33].

Considering the promise of Schema Therapy as a treatment approach for personality disorders [34], present data may have important clinical implications for its implementation in the therapy of depressive symptoms. In line with a view of depression as a multifaceted condition, present findings suggest the existence of specific depressive states in people with different EMSs who experienced an adverse life event. It has to be noted that, in most studies, the EMSs predicted about the $11 \%$ of the variance of the scores at depression inventories [33]; our predictions of each specific depressive symptom were much more powerful (up to $45 \%$ ).

Several limitations need to be acknowledged. The major limit is the cross-sectional nature of the study that precludes any conclusions about the direction of effects. Moreover, the dysphoric episode occurred before the compilation of the YSQ. As a fundamental assumption in schema theory is that EMSs are stable, trait-like constructs that are resistant to change $[5,12]$, also during the occurrence of episodes of depression $[9,10]$, there is no reason to think that this may have biased the results. Third, problems in data interpretation may arise from the use of retrospective self-reports. With regard to generalizability issues, it has to be noted that the sample was self-selected (i.e., willing to participate in an online survey) and consists of a majority of females, but this reflects gender disparities in the prevalence of depressive episodes [35].

Limitations notwithstanding, the current study provides preliminary evidence that specific EMSs may be vulnerability factors for specific depressive symptoms. Future studies on depression in general and on the relationship between EMSs and depression in particular should take into account that several depressive reactions exist based on how individual characteristics, such as EMS, determine the interpretation of adverse life events. Clinical implications are evident if we consider that specific schema domains have been recently shown to predict treatment outcome [27].

\section{Conflict of Interests}

The authors declares that there is no conflict of interests regarding the publication of this paper. 


\section{References}

[1] M. C. Keller, M. C. Neale, and K. S. Kendler, "Association of different adverse life events with distinct patterns of depressive symptoms," The American Journal of Psychiatry, vol. 164, no. 10, pp. 1521-1529, 2007.

[2] M. A. Oquendo, A. Barrera, S. P. Ellis et al., "Instability of symptoms in recurrent major depression: a prospective study," The American Journal of Psychiatry, vol. 161, no. 2, pp. 255-261, 2004.

[3] A. T. Beck, "Thinking and depression. II. Theory and therapy," Archives of General Psychiatry, vol. 10, pp. 561-571, 1964.

[4] A. Couyoumdjian, C. Ottaviani, R. Trincas, G. Spitoni, K. Tenore, and R. Mancini, "The role of personal goals in depressive reaction to adverse life events: a cross-sectional study," The Scientific World Journal, vol. 20122012, Article ID 810341, 8 pages, 2012.

[5] J. E. Young, J. S. Klosko, and M. Weishaar, Schema Therapy: A practitioner's Guide, Guilford Publications, New York, NY, USA, 2003.

[6] J. E. Young, B. Pascal, and P. Cousineau, Young Schema Questionnaire-Short Form 3 (YSQ-S3), Schema Therapy Institute, New York, NY, USA, 2005.

[7] L. D. Hawke and M. D. Provencher, "Schema theory and schema therapy in mood and anxiety disorders: a review," Journal of Cognitive Psychotherapy, vol. 25, no. 4, pp. 257-276, 2011.

[8] S. Bailleux, L. Romo, S. Kindynis, A. Radtchenko, and Q. Debray, "Study of the bonds between early maladaptive schemas and strategies of coping (among alcohol-dependent patients and depressed patients)," Journal de Thérapie Comportementale et Cognitive, vol. 18, no. 1, pp. 19-25, 2008.

[9] L. P. Riso, S. E. Froman, M. Raouf et al., "The long-term stability of early maladaptive schemas," Cognitive Therapy and Research, vol. 30, no. 4, pp. 515-529, 2006.

[10] C. E. A. Wang, M. Halvorsen, M. Eisemann, and K. Waterloo, "Stability of dysfunctional attitudes and early maladaptive schemas: a 9-year follow-up study of clinically depressed subjects," Journal of Behavior Therapy and Experimental Psychiatry, vol. 41, no. 4, pp. 389-396, 2010.

[11] M. Halvorsen, C. E. Wang, J. Richter et al., "Early maladaptive schemas, temperament and character traits in clinically depressed and previously depressed subjects," Clinical Psychology and Psychotherapy, vol. 16, no. 5, pp. 394-407, 2009.

[12] L. Stopa and A. Waters, "The effect of mood on responses to the Young Schema Questionnaire: short form," Psychology and Psychotherapy, vol. 78, no. 1, pp. 45-57, 2005.

[13] E. Calvete, A. Estévez, E. L. de Arroyabe, and P. Ruiz, "The schema questionnaire-short form: structure and relationship with automatic thoughts and symptoms of affective disorders," European Journal of Psychological Assessment, vol. 21, no. 2, pp. 90-99, 2005.

[14] A. E. Harris and L. Curtin, "Parental perceptions, early maladaptive schemas, and depressive symptoms in young adults," Cognitive Therapy and Research, vol. 26, no. 3, pp. 405-416, 2002.

[15] N. B. Schmidt, T. E. Joiner Jr., J. E. Young, and M. J. Telch, “The schema questionnaire: investigation of psychometric properties and the hierarchical structure of a measure of maladaptive schemas," Cognitive Therapy and Research, vol. 19, no. 3, pp. 295321, 1995.

[16] L. Stopa, P. Thorne, A. Waters, and J. Preston, "Are the short and long forms of the Young Schema Questionnaire comparable and how well does each version predict psychopathology scores?" Journal of Cognitive Psychotherapy, vol. 15, no. 3, pp. 253-272, 2001.

[17] K. Welburn, M. Coristine, P. Dagg, A. Pontefract, and S. Jordan, "The schema questionnaire-short form: factor analysis and relationship between schemas and symptoms," Cognitive Therapy and Research, vol. 26, no. 4, pp. 519-530, 2002.

[18] L. P. Riso, J. A. Blandino, S. Penna et al., "Cognitive aspects of chronic depression," Journal of Abnormal Psychology, vol. 112, no. 1, pp. 72-80, 2003.

[19] G. Waller, R. Shah, V. Ohanian, and P. Elliott, "Core beliefs in Bulimia nervosa and depression: the discriminant validity of young's schema Questionnaire," Behavior Therapy, vol. 32, no. 1, pp. 139-153, 2001.

[20] R. M. Nesse, "Why has natural selection left us so vulnerable to anxiety and mood disorders?" Canadian Journal of Psychiatry, vol. 56, no. 12, pp. 705-706, 2011.

[21] A. T. Beck, Depression: Clinical, Experimental, and Theoretical Aspects, Harper and Row, New York, NY, USA, 1967.

[22] G. Waller, V. Ohanian, C. Meyer, and S. Osman, "Cognitive content among bulimic women: the role of core beliefs," International Journal of Eating Disorders, vol. 28, no. 2, pp. 235-241, 2000.

[23] G. A. Fava, "Assessing depressive symptoms across cultures: Italian validation of the CES-D self-rating scale," Journal of Clinical Psychology, vol. 39, no. 2, pp. 249-251, 1983.

[24] M. C. Keller and R. M. Nesse, "Is low mood an adaptation? Evidence for subtypes with symptoms that match precipitants," Journal of Affective Disorders, vol. 86, no. 1, pp. 27-35, 2005.

[25] M. C. Keller and R. M. Nesse, "The evolutionary significance of depressive symptoms: different adverse situations lead to different depressive symptom patterns," Journal of Personality and Social Psychology, vol. 91, no. 2, pp. 316-330, 2006.

[26] A. T. Beck, "Cognitive therapy of depression: new perspectives," in Treatment of Depression: Old Controversies and New Approaches, P. J. Clayton and J. E. Barrett, Eds., Raven Press, New York, NY, USA, 1983.

[27] F. Renner, J. Lobbestael, F. Peeters, A. Arntz, and M. Huibers, "Early maladaptive schemas in depressed patients: stability and relation with depressive symptoms over the course of treatment," Journal of Affective Disorders, vol. 136, no. 3, pp. 581-590, 2012.

[28] M. Ahmadian, L. Fata, A. Asgharnezhad, and K. Malakouti, "A comparison of the early maladaptive schemas of suicidal and non-suicidal depressed patients with non-clinical sample," Advances in Cognitive Science, vol. 10, pp. 49-59, 2009.

[29] L. D. Hawke and M. D. Provencher, "Early Maladaptive Schemas among patients diagnosed with bipolar disorder," Journal of Affective Disorders, vol. 136, no. 3, pp. 803-811, 2012.

[30] M. Halvorsen, C. E. Wang, M. Eisemann, and K. Waterloo, "Dysfunctional attitudes and early maladaptive schemas as predictors of depression: a 9-year follow-up study," Cognitive Therapy and Research, vol. 34, no. 4, pp. 368-379, 2010.

[31] R. Shah and G. Waller, "Parental style and vulnerability to depression: the role of core beliefs," Journal of Nervous and Mental Disease, vol. 188, no. 1, pp. 19-25, 2000.

[32] R. C. Shorey, G. L. Stuart, and S. Anderson, "The early maladaptive schemas of an opioid-dependent sample of treatment seeking young adults: a descriptive investigation," Journal of Substance Abuse Treatment, vol. 42, no. 3, pp. 271-278, 2012. 
[33] S. Nolen-Hoeksema and L. M. Hilt, "Gender differences in depression," in Handbook of Depression, C. Hammen and I. Gotlib, Eds., The Guilford Press, New York, NY, 2009.

[34] J. Giesen-Bloo, R. van Dyck, P. Spinhoven et al., "Outpatient psychotherapy for borderline personality disorder randomized trial of schema-focused therapy vs transference-focused psychotherapy," Archives of General Psychiatry, vol. 63, no. 6, pp. 649-658, 2006.

[35] R. C. Kessler, K. A. McGonagle, M. Swartz, D. G. Blazer, and C. B. Nelson, "Sex and depression in the National Comorbidity Survey I: lifetime prevalence, chronicity and recurrence," Journal of Affective Disorders, vol. 29, no. 2-3, pp. 85-96, 1993. 


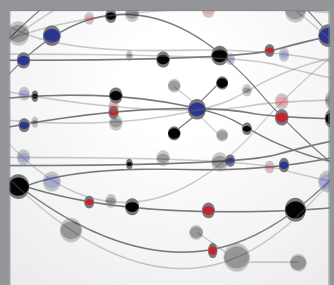

The Scientific World Journal
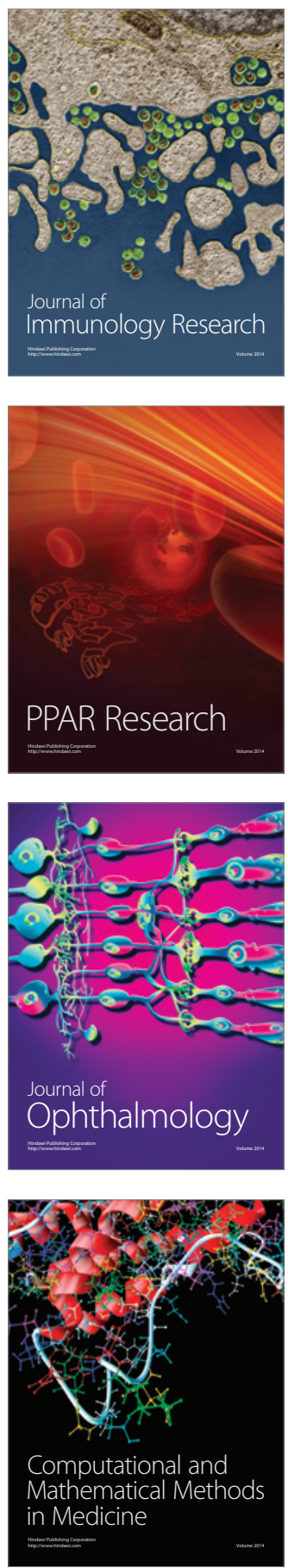

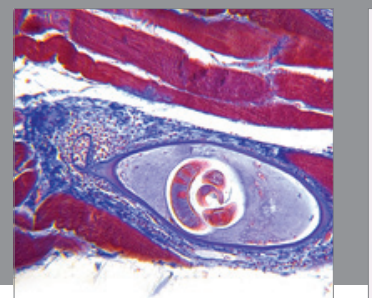

Gastroenterology

Research and Practice
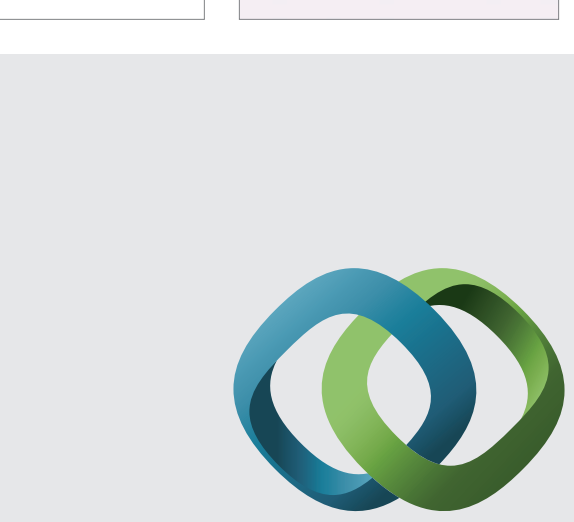

\section{Hindawi}

Submit your manuscripts at

http://www.hindawi.com
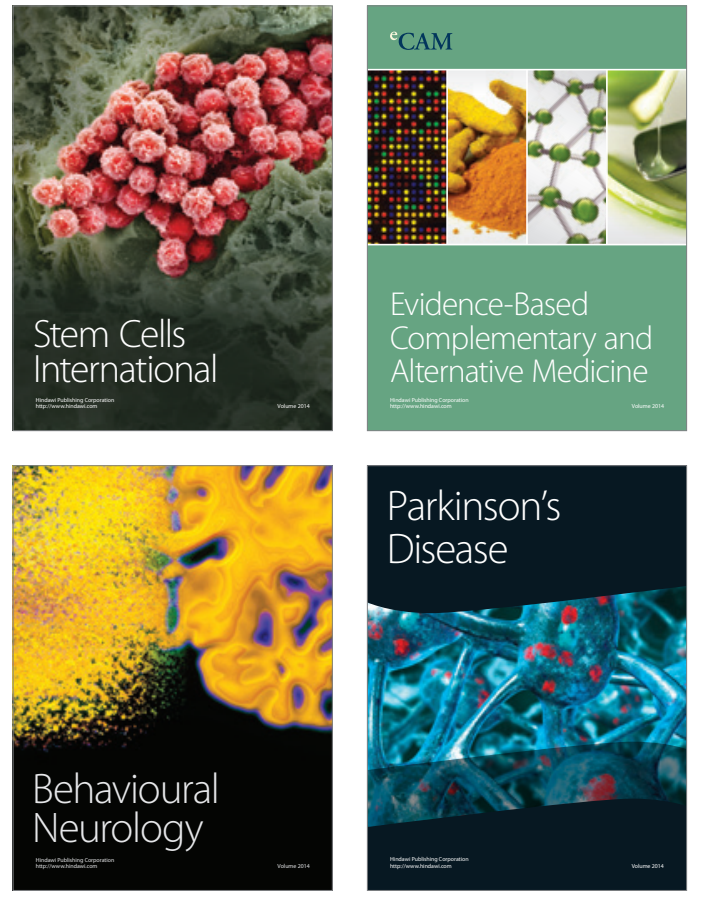
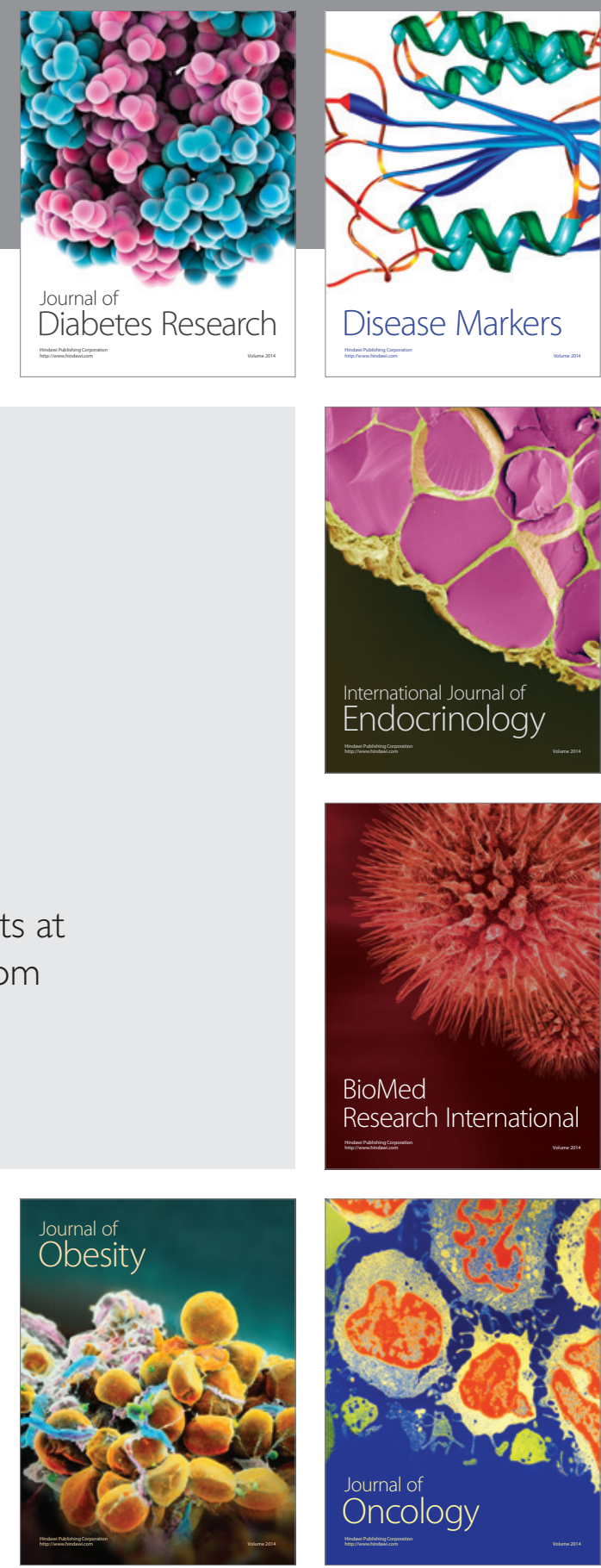

Disease Markers
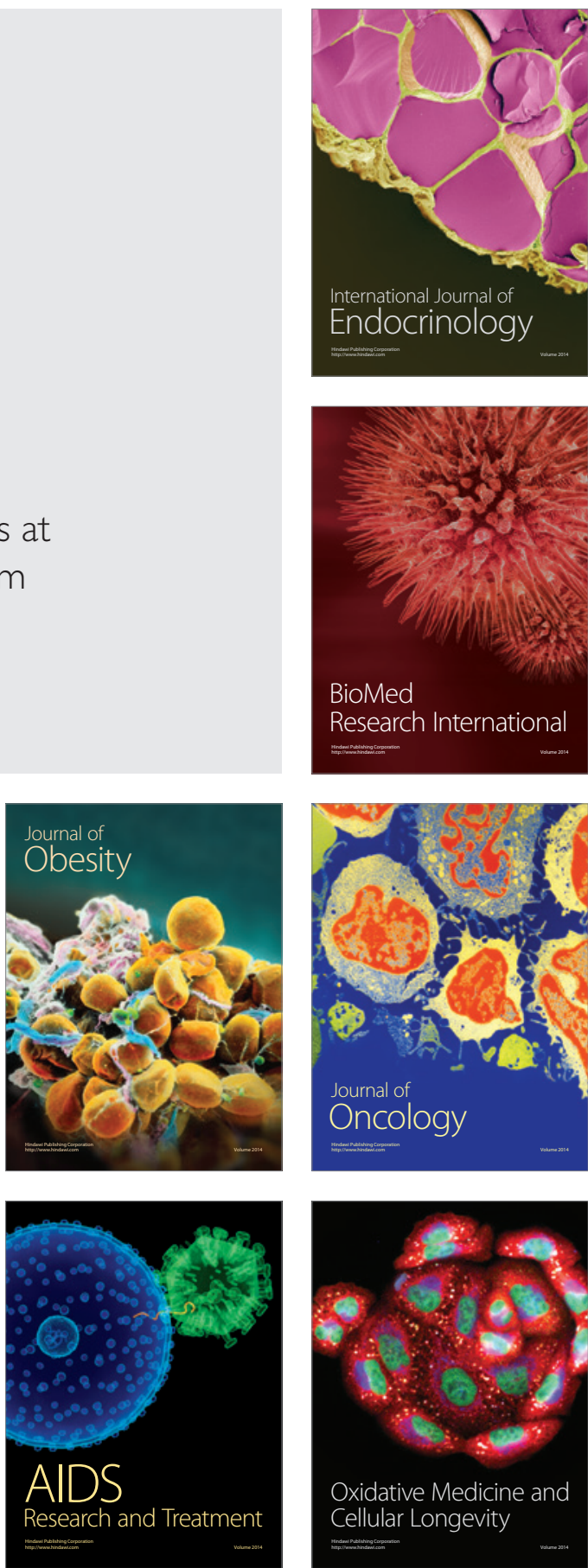